\title{
Structural and Optical Behaviour of Ni Doped CdS Nanoparticles Synthesized by Chemical Co-Precipitation Method
}

\author{
K. Siva Kumar ${ }^{a}$, A. Divya ${ }^{a}$, P. Sreedhara Reddy ${ }^{a}$, S. Uthanna ${ }^{a}$, R. Martins $^{b}$ \\ AND E. ElANGOVAN ${ }^{b, *}$ \\ ${ }^{a}$ Department of Physics, Sri Venkateswara University, Tirupati - 517 502, India \\ ${ }^{b}$ CENIMAT/I3N, Departemento de Ciencia dos Materiais, Faculdade de Cienciase Technolgia, FCT \\ Universidade Nova de Lisboa and CEMOP/UNINOVA, 2829-516 Caparica, Portugal
}

Pure and $\mathrm{Ni}$ (2 at.\%) doped cadmium sulfide (CdS) nanoparticles have been synthesized by chemical co-precipitation method at room temperature. Effect of $\mathrm{Ni}$ doping on CdS compound semiconductors has been analyzed using X-ray diffractometer, transmission electron microscope, energy dispersive spectroscopy, Raman and optical absorption studies. X-ray diffraction study confirmed the structure of the obtained nanoparticles to be single-phase zinc blende with the diffractions from (111), (220) and (311) planes. Energy dispersive spectroscopy study confirmed the presence of $\mathrm{Ni}$ in the CdS lattice. The average particle size obtained from the transmission electron microscopy studies are in agreement with the crystallite size calculated from X-ray diffraction data. The optical studies exhibited a clear red shift of absorption edge as a function of Ni doping.

PACS: 61.05.Cp, 61.72.-y, 78.55.Et, 78.67.Bf

\section{Introduction}

Semiconducting materials with nanometer-scale confinement of electrons provide number of quantum phenomena such as low dimensional electronic states, increased exciton binding energies and dynamics of carriers in the systems. Diluted magnetic semiconductors (DMSs) [1, 2] referred as semiconductors doped with transition metal element or rare earths have attracted much attention due to their potential applications in spintronics [3-5]. Therefore, nanostructured DMSs are expected to show particular magnetooptical effects owing to the confinement of both the electronic and magnetic states. In order to gain advantages offered by the spin, it is necessary to have low dimensional structure to integrate the DMS material into electronic devices. Among the II-VI DMSs, CdS based DMSs have received much attention due to its potential applications such as magnetooptical and future spintronic devices. In recent years, transition metal ion-doped nanoparticles have been studied because of their unique optical properties [6-9] and potential for various applications other than biomedical labeling [10, 11]. Despite these advantages, no systematic investigation on $\mathrm{Ni}$ doped $\mathrm{CdS}$ was carried out. In

\footnotetext{
* corresponding author; e-mail:

eevan2003@yahoo.co.in; elam@fct.unl.pt
}

this report, pristine and $\mathrm{Ni}$ doped $\mathrm{CdS}$ nanoparticles were prepared by chemical co-precipitation method by using polyvnylpyrrolidone (PVP) as a surfactant. The morphological and optical absorption studies on the pure and Ni doped CdS nanoparticles were systematically investigated and the obtained results are interpreted.

\section{Experimental details}

The synthesis of PVP capped pure and Ni (2 at.\%) doped CdS nanoparticles have been prepared at room temperature by chemical co-precipitation. All chemicals were of analytical reagent grade and were used directly without further purification. The deionized water was used as solvent for all the solutions referred in the present work. First, a desired molar proportion of $\mathrm{Cd}\left(\mathrm{CH}_{3} \mathrm{COO}\right)_{2} \cdot 2 \mathrm{H}_{2} \mathrm{O}$ and $\mathrm{NiCl}_{2} \cdot 6 \mathrm{H}_{2} \mathrm{O}$ were dissolved in the deionized water. Then, an appropriate amount of PVP was added to the mixture as a capping agent. After stirring this solution for $60 \mathrm{~min}$, the $\mathrm{pH}$ of the mixture was adjusted to 8.0, and then the thiourea solution was added dropwise with continuous vigorous stirring. Dried powders of CdS:Ni nanoparticles were obtained by precipitation and heating.

The structure of pristine and $\mathrm{Ni}$ (2 at.\%) doped CdS nanoparticles were analyzed by using a Seifert 3003TT X-ray diffractometer (XRD) with $\mathrm{Cu} K_{\alpha}$ radiation $(\lambda=$ $1.54060 \AA)$. The size and composition of the nanoparticles were studied by transmission electron microscopy 
(TEM) of model JEOL 2010 and an Oxford make Inca Penta FeTX3 energy dispersive spectroscopy (EDS) attachment in Carl Zeiss EVO MA 15 SEM instrument. Optical absorption studies were carried out using Carey5E UV-VIS-NIR lambda 950 double-beam spectrophotometer in the wavelength ranging 300-700 $\mathrm{nm}$. The Raman measurements were performed on Renishaw Raman spectrophotometer (model RM 1000 System) using laser with wavelength of $514.5 \mathrm{~nm}$.

\section{Results and discussion}

Figure 1 shows the XRD patterns obtained from pristine and $\mathrm{Ni}$ doped $\mathrm{CdS}$ nanoparticles. The obtained broad diffracted peaks are indicating that the size of crystallites should be in the nanometer scale. The broad diffracted peaks obtained at diffraction angles, $2 \theta$, of $26.49^{\circ}, 43.61^{\circ}$ and $52.23^{\circ}$ are indexed as (111), (220) and (311) by matching with the standard cubic zinc blende phase of CdS (JCPDS file no. 10-454). It is noteworthy that no secondary diffraction peaks from cadmium oxide, nickel oxide, nickel sulfide and elemental sulfur were detected in the XRD patterns. As anticipated, the XRD peaks of undoped and doped CdS samples were significantly broadened when compared with bulk CdS. In addition, the relative intensities of (111), (220) and (311) diffractions were observed to vary in Ni doped CdS nanoparticles. It could be due to the replacement of $\mathrm{Cd}^{2+}$ by $\mathrm{Ni}^{2+}$ at different lattice sites of CdS. The obtained XRD patterns do not show any noticeable shift in the major diffraction peaks and so the undoped CdS crystal structure was retained in all the samples, with the occupancy of $\mathrm{Cd}^{2+}$ by $\mathrm{Ni}^{2+}$. The mean crystalline size of the sample was determined from the full width at half-maximum (FWHM) on the most intense peak making use of the Debye-Scherrer equation [12] and was found to be about $8 \mathrm{~nm}$. To substantiate the size and microstructure of the synthesized nanoparticles, it is obligatory to analyze the product by a direct analysis tool like TEM. A typical TEM image obtained from 2 at.\% Ni doped CdS nanoparticles is shown in Fig. 2. In all cases, the particles were spherical in shape and there was good homogeneity in particle size distribution.

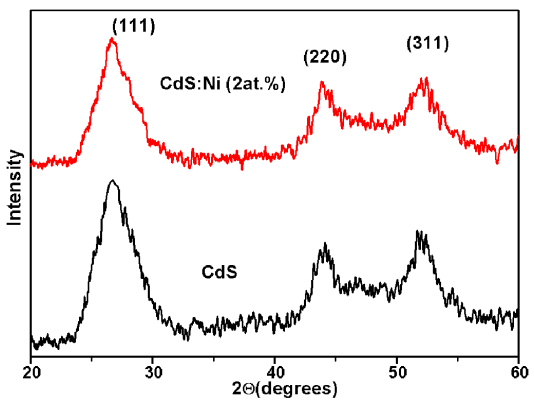

Fig. 1. XRD patterns of pure and Ni (2 at.\%) doped CdS nanoparticles.

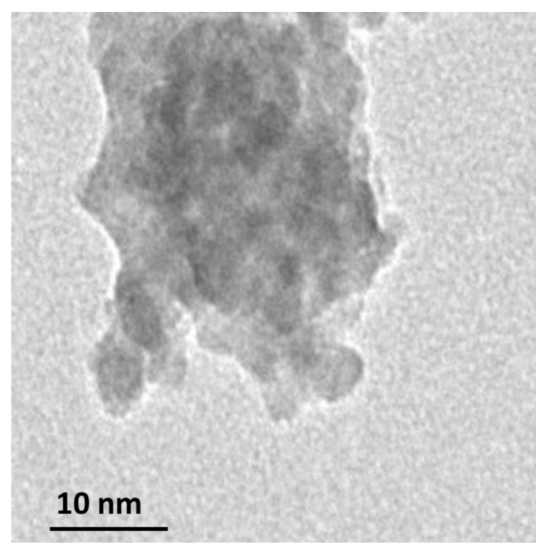

Fig. 2. TEM image of CdS:Ni (2 at.\%) nanoparticles.

The average size of aggregated nanoparticles considering the minimum and maximum diameter with large number of particles were found to be $7 \mathrm{~nm}$. The EDS analysis of CdS:Ni nanoparticles were carried out to establish the purity of the samples and to confirm presence of $\mathrm{Ni}$ content in the samples (Fig. 3). The EDS spectrum was recorded at five different locations on each sample to ascertain the homogeneity of the $\mathrm{Ni}$ incorporation in the CdS nanoparticles. It revealed that $\mathrm{Cd}, \mathrm{Ni}$ and $\mathrm{S}$ elements were present in the sample, which further confirmed the successful doping of $\mathrm{Ni}$ in the host CdS. Thus, the nanoparticles were found to contain no spurious contamination. The ratio of the atomic percentage of the elements present in the samples complied with the quantity taken for their preparation.

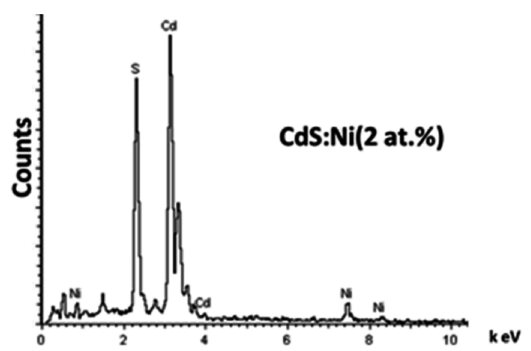

Fig. 3. EDS spectrum of $\mathrm{Ni}(2$ at.\%) doped CdS nanoparticles.

The Raman studies were carried out for the investigation of the changes observed in the atomic arrangement of CdS lattice upon $\mathrm{Ni}^{2+}$ doping. The Raman spectra of the pristine and $\mathrm{Ni}$ doped $\mathrm{CdS}$ nanoparticles are shown in Fig. 4. The Raman peaks at 300 and $601 \mathrm{~cm}^{-1}$ correspond to the $1 \mathrm{LO}$ and $2 \mathrm{LO}$ phonon modes of CdS which were consistent with reported results $[13,14]$ with no other peaks related with the impurities. Compared with these Raman modes of CdS nanoparticles, the Raman modes of $\mathrm{Ni}$ doped $\mathrm{CdS}$ nanoparticles were slightly red shifted as the ionic radius of $\mathrm{Ni}^{2+}$ is less than that of $\mathrm{Cd}^{2+}$. In addition, with $\mathrm{Ni}^{2+}$ doping there is an addi- 


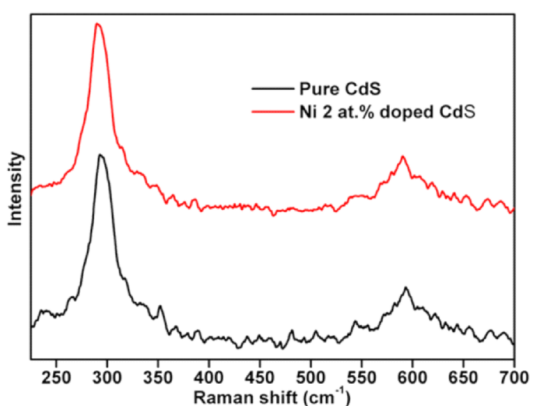

Fig. 4. Raman spectra of pure and Ni (2 at.\%) doped CdS nanoparticles.

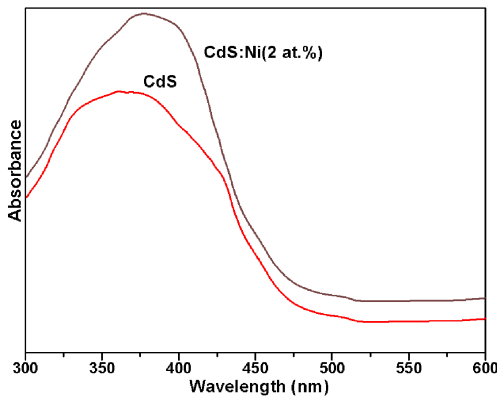

Fig. 5. Optical absorption spectra of pure and $\mathrm{Ni}$ (2 at.\%) doped CdS nanoparticles.

tional factor of lattice softening because $\mathrm{Ni}^{2+}$ replaces the $\mathrm{Cd}^{2+}$ sites, leading to overall shift of the Raman modes to lower phonon energies.

Optical absorption measurements were carried out at room temperature on pure and CdS:Ni nanoparticles, and the obtained spectra are shown in Fig. 5. Pure CdS and Ni doped CdS samples showed absorption band edge at 378 and $398 \mathrm{~nm}$, respectively, which were shifted towards shorter wavelengths from bulk values due to quantum confinement effect. The red shift of band edge for the nickel-doped samples clearly indicated that $\mathrm{Ni}^{2+}$ ions were incorporated into the CdS lattice [15].

\section{Conclusions}

PVP stabilized Ni doped CdS nanoparticles were successfully synthesized at room temperature by chemical co-precipitation technique. The X-diffraction analysis reveals that undoped and Ni-doped CdS nanoparticles exhibited cubic zinc blende structure with (111), (220) and
(311) planes. EDS analysis confirmed that Ni was successfully doped into CdS lattice. TEM results were consistent with those of XRD. The Raman studies revealed that the $1 \mathrm{LO}$ and $2 \mathrm{LO}$ peaks showed red shift compared to undoped $\mathrm{CdS}$ nanoparticles. Absorption edge of $\mathrm{Ni}$ doped CdS nanoparticles showed red shift when compared to pure CdS nanoparticles.

\section{Acknowledgments}

One of the authors (KSK) is grateful to University Grants Commission, New Delhi, India for providing Junior Research Fellowship under UGC-RFSMS scheme.

\section{References}

[1] H. Ohno, Science 281, 951 (1998).

[2] J.K. Furdyna, J. Appl. Phys. 64, R29 (1988).

[3] H. Ohno, A. Shen, F. Matsukura, A. Oiwa, A. Endo, S. Katsumoto, Y. Iye, Appl. Phys. Lett. 69, 363 (1996).

[4] S.A. Wolf, D.D. Awschalom, R.A. Buhrman, J.M. Daughton, S. von Molnar, M.L. Roukes, A.Y. Chtchelkanova, D.M. Treger, Science 294, 1488 (2001).

[5] M. Johnson, J. Phys. Chem. B 109, 14278 (2005).

[6] F.V. Mikulec, M. Kuno, M. Bennati, D.A. Hall, R.G. Griffin, M.G. Bawendi, J. Am. Chem. Soc. 122, $2532(2000)$.

[7] O.E. Raola, G.F. Strouse, Nano Lett. 2, 1443 (2002).

[8] O. Lehmann, K. Kompe, M. Haase, J. Am. Chem. Soc. 126, 14935 (2004).

[9] L. Zu, D.J. Norris, T.A. Kennedy, S.C. Erwin, A.L. Efros, Nano Lett. 6, 15334 (2006).

[10] N.S. Norberg, K.R. Kittilstved, J.E. Amonette, R.K. Kukkadapu, D.A. Schwartz, D.R. Gamelin, J. Am. Chem. Soc. 126, 9387 (2004).

[11] X. Huang, A. Makmal, J.R. Chelikowsky, L. Kronik, Phys. Rev. Lett. 94, 236801 (2005).

[12] B.D. Cullity, Elements of X-ray Diffraction, Addison Wesley, USA 1978.

[13] A. Phuruangrat, T. Thongtem, S. Thongtem, Mater. Lett. 63, 1538 (2009).

[14] T. Thongtem, A. Phuruangrat, S. Thongtem, Ceram. Int. 35, 2817 (2009).

[15] M. Thambidurai, N. Muthukumarasamy, S. Agilan, N. Sabari Arul, N. Murugan, R. Balasundaraprabhu, J. Mater. Sci. 46, 3200 (2010). 